\title{
SPATIALLY PERIODIC, FORCE-FREE MAGNETIC FIELDS WITH RESISTIVE DECAY*
}

\author{
BY \\ RUDOLF X. MEYER \\ The Aerospace Corporation
}

\begin{abstract}
Force-free magnetic fields, i.e. fields that are either parallel or antiparallel to the electric current, occur in the solar chromosphere and also have been used in certain magnetic confinement schemes in controlled thermonuclear fusion research. In this paper, we derive a general expression for force-free fields that decay resistively and are spatially periodic.

The general expression that is found consists of a sum of spatial Fourier modes with the property that all wave vectors have the same absolute magnitude, with components that satisfy a Diophantine equation. A large class of particular solutions is found to exist.

Jette's theorem in resistive magnetohydrostatics is rederived for the case of periodic fields by means of a simple geometrical proof. In the concluding section the results are extended to the case where the electric conductivity is a function of time. An application to the study of iso-energetic Beltrami flows in classical fluid mechanics is indicated.
\end{abstract}

1. Introduction. Force-free magnetic fields, that is, fields for which the Lorentz force $\mathbf{j} \times \mathbf{B}$ ( $\mathbf{j}=$ current density, $\mathbf{B}=$ magnetic induction) vanishes, have been studied by Chandrasekhar and Kendall [1] and Voslamber and Callebaut [2], among others. Since in magnetohydrodynamic applications the displacement current can usually be neglected, the condition for the field to be force-free reduces to

$$
\nabla \times \mathbf{B}=\alpha \mathbf{B}
$$

where $\alpha$ is a scalar that in general is a function of the spatial coordinates and of time. In addition, the field must satisfy the solenoidal condition

$$
\nabla \cdot \mathbf{B}=0 .
$$

Since $\alpha$ is unknown, the force-free condition leads in general to a difficult nonlinear problem.

Most authors therefore have concentrated on the simpler case obtained by considering force-free fields for which $\alpha$ is constant. For this case, Chandrasekhar and Kendall [1] have given a general solution in the form of a sum of a poloidal and a toroidal vector field, and have applied it to force-free fields within spherical boundaries.

\footnotetext{
* Received August 21, 1981. The author wishes to thank Drs. R. H. Huddlestone and D. Pridmore-Brown for critically reading the manuscript and for making a number of suggestions that have been incorporated in this paper.
} 
Since no forces are applied to the fluid (at least in the magnetohydrodynamic approximation), such magnetic field configurations are of interest for stable containment of thermonuclear plasmas. Magnetic fields in the solar chromosphere are approximately forcefree [3], and observed fields have been analyzed on this basis [4].

In a separate class of magnetohydrodynamic problems, time-varying magnetic fields are considered that remain force-free in time. In the simplest such problems, constant electric conductivity $\sigma$ and a negligibly small magnetic Reynolds number are assumed. The magnetic field then must in addition satisfy the vector diffusion equation

$$
\partial \mathbf{B} / \partial t=\frac{1}{\mu \sigma} \nabla^{2} \mathbf{B} .
$$

Eqs. (1) to (3) are the basic equations for the remainder of this paper.

Lundquist [5] conjectured that the only magnetic fields that stay force-free in time are those corresponding to constant $\alpha$. A proof of this theorem was provided by Jette [6]. The proof is extremely lengthy and assumes inter alia that spatial derivatives of $\alpha$ exist up to the third derivative. In the course of deriving a general representation for spatially periodic fields satisfying (1) to (3), the present paper rederives Jette's result with a much shorter proof restricted now to periodic fields. Only the existence of the first derivative of $\alpha$ needs to be assumed.

A simple consequence of (1) to (3) is obtained by making use of the solenoidal condition and substituting twice from (1) into (3), with the result that

$$
\partial \mathbf{B} / \partial t=-\frac{1}{\mu \sigma} \nabla \times(\nabla \times \mathbf{B})=-\frac{1}{\mu \sigma}(\nabla \alpha \times \mathbf{B}+\alpha \nabla \times \mathbf{B})=-\frac{1}{\mu \sigma}\left(\alpha^{2} \mathbf{B}+\nabla \alpha \times \mathbf{B}\right) .
$$

This result will be needed later. We note that in the special case where $\alpha$ is constant, we have the important physical consequence that the magnetic field at any point does not change direction as it decays with time.

2. Normal mode expansion. We proceed by expanding the magnetic field in terms of normal modes which satisfy the vector diffusion equation and the requirement that the field be solenoidal. After having thus satisfied the set of conditions that are linear, the nonlinear force-free field condition is satisfied by substituting into it the normal mode expansion. This results in simple algebraic relations among the expansion coefficients. An inequality governing the wave numbers then leads to the desired result.

It will be found advantageous to use the notation of complex vector spaces. Thus, the inner product $(\mathbf{p} \cdot \mathbf{q})$ of two vectors in an $n$-dimensional space is defined as $\sum_{i=1}^{n} p_{i} q_{i}^{*}$ where ( $)^{*}$ indicates the complex conjugate. Hence, $(\mathbf{p} \cdot \mathbf{q})=(\mathbf{q} \cdot \mathbf{p})^{*}$. The vector product in three-dimensional space is defined in terms of components in the same way as it is for real vectors. We will also need to use the expansion for the triple vector product of complex vectors, $\mathbf{p} \times(\mathbf{q} \times \mathbf{r})=\left(\mathbf{p} \cdot \mathbf{r}^{*}\right) \mathbf{q}-\left(\mathbf{p} \cdot \mathbf{q}^{*}\right) \mathbf{r}$.

It is convenient to take the radius vector as $a \rho$ where $a$ is a constant length characterizing the spatial scale of the magnetic field, and to let the time $t=\mu \sigma a^{2} \tau$, where $\rho$ and $\tau$ are dimensionless.

Eq. (3) is equivalent to the set of scalar diffusion equations, separately for each of the three Cartesian components. As is well known [7], a complete set of normal modes of the diffusion equation can therefore be constructed by separation of variables, with the result 
that the general solution of (3) can be written in the form

$$
\mathbf{B}=\sum_{\mathbf{k}} \mathbf{b}(\mathbf{k}) \exp \left(i \mathbf{k} \cdot \boldsymbol{\rho}-k^{2} \tau\right)
$$

where the wave vector $\mathbf{k}$ is a vector in a real, three-dimensional vector space and where $\mathbf{k}$ is integer-valued (i.e., has integer-valued components). The summation is a triple sum extended over all values of the components of $\mathbf{k}$. All vectors in this equation are real, with the exception of the expansion coefficients $\mathbf{b}(\mathbf{k})$ which are in general complex. To assure that the magnetic field $\mathbf{B}$ is real, it is required that

$$
\mathbf{b}(-\mathbf{k})=\mathbf{b}^{*}(\mathbf{k}) \text {. }
$$

Substitution of the expansion (5) into the solenoidal condition (2) yields the relation

$$
\mathbf{b}(\mathbf{k}) \cdot \mathbf{k}=\mathbf{0} \text {. }
$$

We write the force-free condition (1) equivalently as $(\nabla \times \mathbf{B}) \times \mathbf{B}=0$. Substitution of the expansion (5) into this equation results in the following nonlinear relation among the expansion coefficients:

$$
\sum_{\mathbf{k}, \mathbf{k},}(\mathbf{k} \times \mathbf{b}(\mathbf{k})) \times \mathbf{b}\left(\mathbf{k}^{\prime}\right) \exp \left[i\left(\mathbf{k}+\mathbf{k}^{\prime}\right) \cdot \mathbf{\rho}-\left(k^{2}+k^{\prime 2}\right) \tau\right]=0,
$$

where the summation symbol indicates a six-fold summation over all integer values of the components of $\mathbf{k}$ and $\mathbf{k}^{\prime}$.

LEMMA 1. For all non-zero, integer values of $\mathbf{k}$,

$$
\mathbf{b}(\mathbf{k}) \cdot \mathbf{b}^{*}(\mathbf{k})=0, \quad k \neq 0
$$

(for the convention used in defining the scalar product, see second paragraph of this section).

Proof: Let $\mathbf{j}$ be a vector with integer components in a three-dimensional, real vector space (the same space as for $\mathbf{k}$ ). The wave vectors that produce terms in (8) with the spatial and temporal dependence $\exp \left[2\left(i j \cdot \rho-j^{2} \tau\right)\right]$ must satisfy the conditions

$$
\mathbf{k}+\mathbf{k}^{\prime}=2 \mathbf{j}, \quad k^{2}+k^{\prime 2}=2 j^{2} .
$$

Clearly, $\mathbf{k}=\mathbf{k}^{\prime}=\mathbf{j}$ is a solution. It is the only solution, for substitution of the two conditions into the identity $k^{2}+k^{\prime 2}=\frac{1}{2}\left(\mathbf{k}+\mathbf{k}^{\prime}\right)^{2}+\frac{1}{2}\left(\mathbf{k}-\mathbf{k}^{\prime}\right)^{2}$ shows that $\left(\mathbf{k}-\mathbf{k}^{\prime}\right)^{2}=0$, hence $\mathbf{k}=\mathbf{k}^{\prime}=\mathbf{j}$. Therefore, there is only one term in (8) with this spatial and temporal dependence. Since it must vanish,

$$
(\mathbf{k} \times \mathbf{b}) \times \mathbf{b}=(\mathbf{b} \cdot \mathbf{k}) \mathbf{b}-\left(\mathbf{b} \cdot \mathbf{b}^{*}\right) \mathbf{k}=\mathbf{0}
$$

where we write $\mathbf{b}$ for $\mathbf{b}(\mathbf{k})$. The lemma then follows by making use of (7).

As a corollary, we can derive the following relation among the expansion coefficients. Thus, in the vector identity

$$
\mathbf{b} \times(\mathbf{k} \times \mathbf{b})=\left(\mathbf{b} \cdot \mathbf{b}^{*}\right) \mathbf{k}-(\mathbf{b} \cdot \mathbf{k}) \mathbf{b}
$$

it follows from (9) and (7) that the right-hand side vanishes. Hence $\mathbf{k} \times \mathbf{b}=\beta \mathbf{b}$ where $\beta=\beta(\mathbf{k})$ is a scalar. On the other hand, from the identity

$$
(\mathbf{k} \times \mathbf{b}) \cdot(\mathbf{k} \times \mathbf{b})=k^{2}(\mathbf{b} \cdot \mathbf{b})-(\mathbf{k} \cdot \mathbf{b})\left(\mathbf{k} \cdot \mathbf{b}^{*}\right)
$$


and (7) follows $\beta \beta^{*}=k^{2}$. Hence, $\beta=k e^{i \phi}$, where the phase angle $\phi$ in general depends on k. Therefore

$$
\mathbf{k} \times \mathbf{b}=k e^{i \phi} \mathbf{b}
$$

In component form this corresponds to three linear, homogeneous equations for the Cartesian components of $\mathbf{b}$. The determinant of the coefficients is $-k^{3}\left(e^{3 i \phi}+e^{i \phi}\right)$. For $\mathbf{b} \neq 0$ and $\mathbf{k} \neq 0$, it follows that $e^{i \phi}= \pm i$; hence,

$$
\mathbf{k} \times \mathbf{b}(\mathbf{k})= \pm i k \mathbf{b}(\mathbf{k})
$$

where the sign in general depends on $\mathbf{k}$. Clearly, this relation holds also for $\mathbf{b}=0$ or $\mathbf{k}=0$.

Substitution into (8) results in the following, simpler form for the force-free field condition:

$$
\sum_{\mathbf{k}, \mathbf{k}^{\prime}} \pm k \mathbf{b}(\mathbf{k}) \times \mathbf{b}\left(\mathbf{k}^{\prime}\right) \exp \left[i\left(\mathbf{k}+\mathbf{k}^{\prime}\right) \cdot \boldsymbol{\rho}-\left(k^{2}+k^{\prime 2}\right) \tau\right]=0 .
$$

3. Relation among wave vectors. Let $k_{1} \geq 0$ be the smallest among the absolute magnitudes of those wave vectors $\mathbf{k}$ for which $\mathbf{b}(\mathbf{k}) \neq 0$, and $k_{2}>k_{1}$ the next smallest such wave number. Excluding the trivial case $\mathbf{B} \equiv 0$, there exists at least one such wave vector of magnitude $k_{1}$.

We consider in (11) all summands that depend on $\rho$ and $\tau$ as

$$
\exp \left[i\left(\mathbf{k}_{1}+\mathbf{k}_{\mathbf{2}}\right) \cdot \boldsymbol{\rho}-\left(k_{1}^{2}+k_{2}^{2}\right) \tau\right] \text {. }
$$

Since, clearly, $k_{1}^{2}+k_{2}^{2}<k^{2}+k^{\prime 2}$ if $k>k_{2}$ or $k^{\prime}>k_{2}$, the only summands with the required time dependence are those that result from taking the summation indexes in (11) such that the magnitude of one index is $k_{1}$, and $k_{2}$ for the other. A pair of wave vectors $\left\{\mathbf{k}_{\mathbf{1}}, \mathbf{k}_{\mathbf{2}}\right\}$ occurring in (11) will be called simple if no other pair with the same magnitudes $k_{1}$ and $k_{2}$ in (11) have the same sum $\mathbf{k}_{1}+\mathbf{k}_{2}$.

Lemma 2. For every wave vector $\mathbf{k}_{\mathbf{2}}$ of magnitude $k_{2}$ and $\mathbf{b}\left(\mathbf{k}_{\mathbf{2}}\right) \neq 0$, there exists a wave vector $\mathbf{k}_{\mathbf{1}}$ with magnitude $k_{1}$ and $\mathbf{b}\left(\mathbf{k}_{\mathbf{1}}\right) \neq 0$ such that the pair $\left\{\mathbf{k}_{\mathbf{1}}, \mathbf{k}_{\mathbf{2}}\right\}$ is simple.

The lemma depends on nothing more than the geometry of a discrete vector space. Fig. 1 illustrates the notation used.

Proof: For a given $\mathbf{k}_{\mathbf{2}}$ define a sequence of pairs $\left\{\mathbf{k}_{1, \mathbf{i}}, \mathbf{k}_{\mathbf{2}, \mathbf{i}}\right\}, i=0,1,2, \ldots$, recursively as follows: (1) choose $\mathbf{k}_{\mathbf{1}, \mathbf{0}}$ arbitrarily among the $\mathbf{k}_{\mathbf{1}}$ vectors and take $\mathbf{k}_{\mathbf{2}, \mathbf{0}}=\mathbf{k}_{\mathbf{2}}$, (2) choose the wave vector pair $\left\{\mathbf{k}_{1, \mathbf{i}+1}, \mathbf{k}_{\mathbf{2}, \mathbf{1}+\mathbf{1}}\right\}$ arbitrarily among those pairs that satisfy the conditon

$$
k_{1, i+1}+k_{2, i+1}=k_{1, i}+k_{2} \text { with } k_{1, i+1} \neq k_{1, i}
$$

if such a pair exists (otherwise the sequence terminates).

To show that this sequence must necessarily terminate, let $\mathbf{h}_{\mathbf{i}}=\mathbf{k}_{\mathbf{2}}-\mathbf{k}_{\mathbf{1}, \mathbf{i}}$. Hence

$$
h_{i}^{2}-h_{i+1}^{2}=2 \mathbf{k}_{\mathbf{2}} \cdot\left(\mathbf{k}_{1, \mathrm{i}+1}-\mathbf{k}_{\mathbf{1}, \mathrm{i}}\right)=2\left(k_{2}^{2}-\mathbf{k}_{\mathbf{2}} \cdot \mathbf{k}_{\mathbf{2}, \mathrm{i}+\mathbf{1}}\right)
$$

from (12). From the Cauchy-Schwarz inequality, $\left|\mathbf{k}_{\mathbf{2}} \cdot \mathbf{k}_{\mathbf{2}, \mathbf{i}+\mathbf{1}}\right| \leq k_{2}^{2}$. Hence, $h_{i}^{2}-h_{i+1}^{2} \geq 0$ and $h_{i} \geq h_{i+1}$.

It follows from (13) that $h_{i}=h_{i+1}$ is the case only when $\mathbf{k}_{\mathbf{2}, \mathbf{i + 1}}=\mathbf{k}_{\mathbf{2}}$ and, hence, from (12), $\mathbf{k}_{\mathbf{1}, \mathbf{i}+\mathbf{1}}=\mathbf{k}_{\mathbf{1}, \mathbf{i}}$. These are just the conditions for which the step by step procedure 
terminates. Hence, the strict inequality

$$
h_{0}>h_{1}>h_{2}, \ldots,>h_{n}
$$

applies. Since there is only a finite number of $\mathbf{k}_{1}$ wave vectors, this sequence must terminate, where the last term $\mathbf{h}_{\mathbf{n}}=\mathbf{k}_{\mathbf{2}}-\mathbf{k}_{\mathbf{1}, \mathbf{n}}$, and where the pair $\left\{\mathbf{k}_{\mathbf{1 , n}}, \mathbf{k}_{\mathbf{2}}\right\}$ is simple.

The inequality (14) has a simple geometric interpretation (Fig. 1). All wave vectors that satisfy (12) terminate on a sphere $S_{i}$ in the wave vector space, with center at $\frac{1}{2}\left(\mathbf{k}_{\mathbf{1}, \mathbf{i}}+\mathbf{k}_{\mathbf{2}}\right)$. Since the length of a chord not a diameter of $S_{i}$ is less than the diameter, the inequality follows.

Henceforth, to simplify the notation, $\mathbf{k}_{\mathbf{1}}$ will stand for $\mathbf{k}_{\mathbf{1}, \mathbf{n}}$. Also, $\mathbf{b}_{\mathbf{1}}$ will stand for $\mathbf{b}\left(\mathbf{k}_{\mathbf{1}}\right)=\mathbf{b}\left(\mathbf{k}_{\mathbf{1}, \mathbf{n}}\right)$ and $\mathbf{b}_{\mathbf{2}}$ for $\mathbf{b}\left(\mathbf{k}_{\mathbf{2}}\right)$.

4. Constancy of $\alpha$. There are, at most, two non-vanishing terms in (11) with the spatial and temporal dependence of the simple pair $\left\{\mathbf{k}_{1}, \mathbf{k}_{\mathbf{2}}\right\}$. Hence, $\left(k_{2} \pm k_{1}\right) \mathbf{b}_{1} \times \mathbf{b}_{\mathbf{2}}=0$. Since, by assumption, $k_{2}>k_{1}$,

$$
b_{1} \times b_{2}=0
$$

We make use of the vector identities, valid for complex $\mathbf{b}_{1}$ and $\mathbf{b}_{\mathbf{2}}$ :

$$
\begin{aligned}
& \mathbf{k}_{1} \times\left(\mathbf{b}_{1} \times \mathbf{b}_{2}\right)=\left(\mathbf{k}_{1} \cdot \mathbf{b}_{2}^{*}\right) \mathbf{b}_{1}-\left(\mathbf{k}_{1} \cdot \mathbf{b}_{1}^{*}\right) \mathbf{b}_{2}, \\
& \mathbf{b}_{2} \times\left(\mathbf{k}_{1} \times \mathbf{k}_{2}\right)=\left(\mathbf{b}_{2} \cdot \mathbf{k}_{2}\right) \mathbf{k}_{1}-\left(\mathbf{b}_{2} \cdot \mathbf{k}_{1}\right) \mathbf{k}_{2} .
\end{aligned}
$$

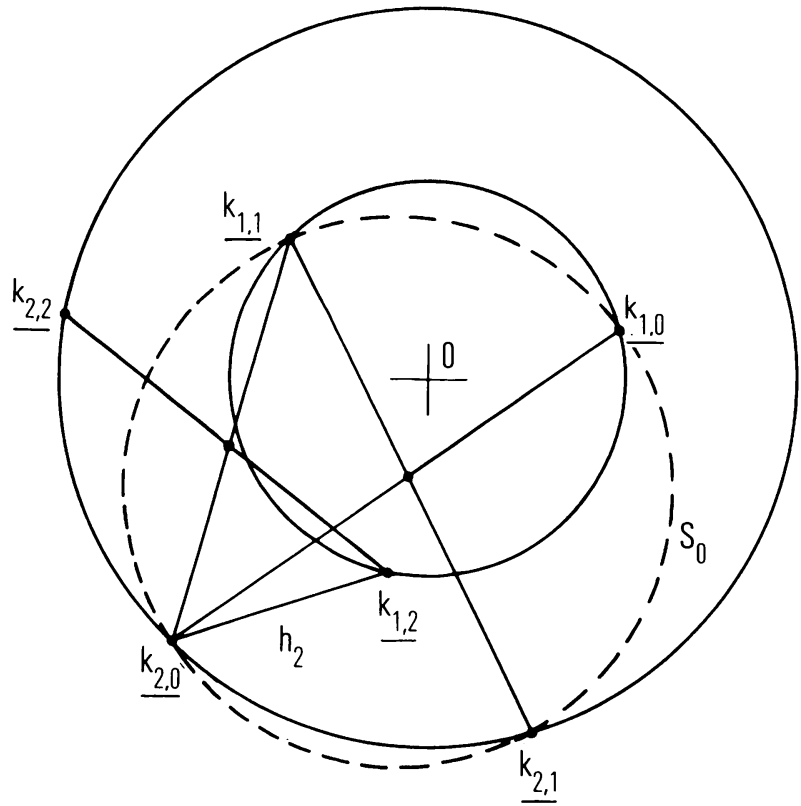

Fig. 1. Illustrates inequality (14) for the case where all wave vectors are coplanar, and where $n=2$. In the general case (wave vectors not necessarily coplanar), wave vectors that satisfy (12) terminate on spheres $S_{i}$ (shown for $i=0$ ). 
From the first identity, with (15) and (7), $\mathbf{k}_{\mathbf{1}} \cdot \mathbf{b}_{\mathbf{2}}^{*}=0$ since $\mathbf{b}_{\mathbf{1}} \neq 0$ by assumption. Hence, from the second identity and (7).

$$
\mathbf{b}_{2} \times\left(\mathbf{k}_{1} \times \mathbf{k}_{2}\right)=0 \text {. }
$$

Case (i): $\mathbf{k}_{\mathbf{1}} \times \mathbf{k}_{\mathbf{2}} \neq 0$. With $\mathbf{b}_{2}=\gamma\left(\mathbf{k}_{1} \times \mathbf{k}_{\mathbf{2}}\right)$, where $\gamma$ is a scalar, it follows from Lemma 1 that $\gamma^{2}\left(\mathbf{k}_{\mathbf{1}} \times \mathbf{k}_{\mathbf{2}}\right)^{2}=0$. Hence, $\gamma=0$ and $\mathbf{b}_{\mathbf{2}}=0$.

Case (ii): $\mathbf{k}_{1} \times \mathbf{k}_{\mathbf{2}}=0$. It follows easily from an application of the Cauchy-Schwarz inequality that the pair $\left\{-\mathbf{k}_{\mathbf{1}}, \mathbf{k}_{\mathbf{2}}\right\}$ in this case is simple also, and that therefore from (11) and (6), $\left(k_{2} \pm k_{1}\right) \mathbf{b}_{1}^{*} \times \mathbf{b}_{2}=0$. Since $k_{2}>k_{1}$, therefore $\mathbf{b}_{1}^{*} \times \mathbf{b}_{2}=0$. Hence $\mathbf{b}_{2}=\delta \mathbf{b}_{\mathbf{1}}^{*}=\varepsilon \mathbf{b}_{1}$ from (15), where $\delta$ and $\varepsilon$ are scalars. From (9) follows $0=\mathbf{b}_{2} \cdot \mathbf{b}_{2}^{*}=\left(\varepsilon \mathbf{b}_{1}\right) \cdot\left(\delta^{*} \mathbf{b}_{1}\right)=\varepsilon \delta \mathbf{b}_{1}^{2}$, where $\mathbf{b}_{1} \neq 0$. Hence, in this case also, $\mathbf{b}_{2}=0$.

Hence there are no non-vanishing modes with wave number $k_{2}$, and therefore it follows from the definition of $k_{2}$ as the wave number next larger than $k_{1}$ that there are no nonvanishing modes other than those with wave number $k_{1}$. The expansion (5) therefore assumes the form

Hence

$$
\mathbf{B}=e^{-k_{1}^{2} \tau} \sum_{\mathbf{k}_{1}} \mathbf{b}\left(\mathbf{k}_{\mathbf{1}}\right) e^{i \mathbf{k}_{1} \cdot \mathbf{p}} .
$$

$$
\partial \mathbf{B} / \partial \tau=-k_{1}^{2} \mathbf{B}
$$

and from (4), taking the component parallel to the local magnetic field vector,

$$
\alpha= \pm k_{1} / a \text {. }
$$

Hence $\alpha$ is constant. (In any vector field determined by (1) to (3), the sign of $\alpha \neq 0$ must stay fixed; in the contrary case $\nabla^{2} \mathbf{B}$ or $\partial \mathbf{B} / \partial \tau$ or both would not be defined.)

That $\alpha$ must be constant for force-free fields which stay force-free in time was first demonstrated by Jette [6]. Jette's proof does not require the vector field to be spatially periodic, but assumes the existence of the third derivative of $\alpha$. His proof, which is by contradiction, is extremely long.

5. General expansion for spatially periodic force-free fields. Eq. (17), together with the auxiliary conditions (6), (7) and (10) for the complex amplitudes b, represents a general expansion for spatially periodic force-free fields. Substitution of these relations into the basic equations (1) to (3) shows that these latter equations are satisfied. (The rank of the coefficient matrix for the auxiliary conditions, when expressed in terms of Cartesian coordinates, is easily shown to be at most 2; thus there exist nontrivial solutions. $\mathbf{B}$ is assured to be real by taking opposite signs in (10), depending on the relation being applied to $\mathbf{b}(\mathbf{k})$ or $\mathbf{b}(-\mathbf{k})$ respectively.) Hence (17), together with (7) and (10), constitutes a necessary and sufficient conditon for the basic equations to hold.

The summation in (17) extends over all wave vectors with components $k_{x}, k_{y}, k_{z}$ that are integer and that satisfy the Diophantine equation

$$
k_{x}^{2}+k_{y}^{2}+k_{z}^{2}=k_{1}^{2} \text {. }
$$

Depending on $k_{1}^{2}$, this equation may have no solution (e.g., for $k_{1}^{2}=7$ ) or many solutions (e.g., for $k_{1}^{2}=41$, where the solutions are $\left(k_{x}, k_{y}, k_{z}\right)=(0, \pm 4, \pm 5),( \pm 1, \pm 2, \pm 6),( \pm 3$, $\pm 4, \pm 4$ ), and permutations of the components in these triplets). 
It follows from (17) that the force-free fields decay exponentially with a characteristic time $\mu \sigma a^{2} / k_{1}^{2}$, and in such a way as to stay self-similar.

The results are easily extended to the case where the conductivity $\sigma=\sigma(t)$ is a function of time. For, if we let

$$
t^{\prime}=\sigma_{0} \int \frac{d t}{\sigma(t)}
$$

the diffusion equation for the magnetic field becomes

$$
\frac{\partial \mathbf{B}}{\partial t^{\prime}}=\frac{1}{\mu \sigma_{0}} \nabla^{2} \mathbf{B}
$$

and hence the same equation as (3). Therefore, by replacing $t$ by $t^{\prime}$, all earlier results still hold and apply now to the case of a time-dependent but spatially constant conductivity.

6. Application to Beltrami flows. The results can also be applied to the study of Beltrami flows [8] in classical fluid mechanics. The incompressible flow of a fluid with linear stress-deformation relation and constant viscosity is described by the Navier-Stokes equation and the conservation of mass condition; therefore

$$
\begin{aligned}
\frac{\partial \mathbf{v}}{\partial t}+(\nabla \times \mathbf{v}) \times \mathbf{v} & =-\nabla H+\nu \nabla^{2} \mathbf{v}, \\
\nabla \cdot \mathbf{v} & =0,
\end{aligned}
$$

where $H=\frac{1}{2} v^{2}+p / \rho+\Omega$. ( $\Omega$ is the potential of the external forces; the meaning of the other symbols is conventional.)

Beltrami flows satisfy the relation

$$
\nabla \times \mathbf{v}=\alpha \mathbf{v}
$$

where $\alpha$ is a scalar. If the flow is isoenergetic, i.e., $H=$ constant, the Navier-Stokes equation then reduces to the diffusion equation

$$
\partial \mathbf{v} / \partial t=v \nabla^{2} \mathbf{v}
$$

Since the governing equations are the same as those for a resistively decaying force-free magnetic field, it follows that the only isoenergetic Beltrami flows of an incompressible, viscous fluid are those for which the vorticity is a constant scalar multiple of the velocity, and where the direction of the local velocity vector is independent of time.

\section{REFERENCES}

[1] S. Chandrasekhar and P. C. Kendall, On force-free magnetic fields, Astrophys. J. 126, 457-460 (1957)

[2] D. Voslamber and D. K. Callebaut, Stability of force-free magnetic fields, Phys. Rev. 128, 2016 (1961)

[3] A. B. Severnyi, Magnetic fields at various depths in the solar atmosphere, Astronomicheskii Zhurnal 43, 465-479 (1966), translated in Soviet Astronomy AJ. 10(3) (1966)

[4] Y. Nakagawa, M. A. Raadu, D. E. Billings, and D. McNamara, On the topology of filaments and chromospheric fibrils near sunspots, Solar Physics 19, 72-85 (1971)

[5] S. Lundquist, Magneto-hydrostatic fields, Arkiv Fysik 2, 361 (1950)

[6] A. D. Jette, Force-free magnetic fields in resistive magnetohydrostatics, J. Math. Anal. Appl. 29, 109-122 
(1970). See also A. D. Jeete and S. R. Sreenivasan, Resistive force-free magnetic fields, Phys. Fluids 12, 2544-2547 (1969)

[7] R. Courant and D. Hilbert, Methods of mathematical physics, vol. 2, Interscience Publishers, New York, 1962 , p. 199

[8] J. Serrin, Mathematical principles of classical fluid mechanics in Handbuch der Physik, S. Flügge, Ed., Vol. VIII/1, Springer, Berlin, 1959, p. 43 